\title{
Communicable Diseases Report, NSW, November and December 2012
}

\author{
Communicable Diseases Branch \\ Health Protection NSW
}

For updated information, including data and facts on specific diseases, visit www.health.nsw.gov.au and click on Public Health and then Infectious Diseases. The communicable diseases site is available at: http://www.health.nsw.gov.au/ publichealth/infectious/index.asp.

Figure 1 and Tables 1 and 2 show notifications of communicable diseases with onset in November and December 2012 in New South Wales (NSW).

\section{Enteric infections \\ Outbreaks of suspected foodborne disease}

There were seven outbreaks of suspected foodborne disease reported in NSW in the period (two in November and five in December), affecting at least 59 people. All outbreaks were thought to be caused by contaminated restaurant food. Of the seven outbreaks, three were reported directly to Public Health Units by the treating doctors or affected individuals, three were reported to the NSW Food Authority and one was identified from an investigation of Salmonella Singapore notifications clustered in time and location.

S. Singapore, $S$. Typhimurium and Clostridium perfringens caused illness in one outbreak each. However, despite thorough case interviews, the food vehicles could not be identified in these outbreaks as cases consumed multiple foods in each instance and no leftover foods were available for testing. In the remaining four outbreaks, the causative organism could not be identified due to either lack of stool collection from ill people and/or lack of sampling of suspected food vehicles.

\section{Viral gastrointestinal disease}

There were 115 outbreaks of gastroenteritis in institutions reported in NSW in the period (72 in November and 43 in December), affecting at least 1735 people. The previous 5-year average for this period was 51 outbreaks.
Of the 115 outbreaks:

- 52 outbreaks (45\%) occurred in aged-care facilities; $41(79 \%)$ of these had one or more stool samples collected - norovirus was confirmed in 17 (41\%) outbreaks and rotavirus was confirmed in one $(2 \%)$ outbreak.

- 46 outbreaks $(40 \%)$ occurred in child-care centres; 7 $(15 \%)$ of these had one or more stool samples collectedrotavirus was confirmed in one (14\%) outbreak.

- 15 outbreaks (13\%) occurred in hospitals; of these, one or more stool samples were collected in 14 (93\%) outbreaks - norovirus was confirmed in $11(79 \%)$ outbreaks and rotavirus was confirmed in one $(7 \%)$ outbreak.

- Two outbreaks $(2 \%)$ occurred in other health facilities and no stool samples were collected.

A stool specimen was collected in 62 outbreaks (54\%); of these, no agent was identified in 32 outbreaks (52\%).

\section{Respiratory infections \\ Influenza}

Influenza, as measured by the number of people who presented with influenza-like illness to 59 of the state's largest emergency departments, continued to circulate at low levels in NSW during November and December 2012. In addition, the number of people who tested positive for influenza A by diagnostic laboratories decreased to preseasonal levels throughout November and December after a peak in late June.

In November, there were:

- 68 presentations to emergency departments (rate 0.4 per 1000 presentations)

- 70 cases of laboratory-confirmed influenza including:

$$
\text { ○ } 14(20 \%) \text { influenza A }
$$$$
\text { ○ } 56(80 \%) \text { influenza B. }
$$

In December, there were:

- 67 presentations to emergency departments (rate 0.4 per 1000 presentations)

- 45 cases of laboratory-confirmed influenza including: - $25(56 \%)$ influenza A

○ $20(44 \%)$ influenza B.

For a more detailed report on respiratory activity in NSW see: http:/www.health.nsw.gov.au/PublicHealth/Infectious/ influenza_reports.asp. 


\section{Vaccine-preventable diseases \\ Meningococcal disease}

Five cases of meningococcal disease were notified in NSW in the period (three in November and two in December), a decrease from nine notified for the same period in 2011. The age of the cases ranged from 2 to 86 years; only one case was aged less than 5 years. An elderly woman from Hunter New England Local Health District died due to meningococcal B infection during this period.

Of the five cases, three $(60 \%)$ were due to serogroup B (for which there is no vaccine), one (20\%) was due to serogroup C, and one (20\%) was due to serogroup Y. The notification of invasive meningococcal disease caused by serogroup $\mathrm{C}$ was in a 26-year old male traveller who had recently arrived from Europe.

Immunisation against meningococcal $\mathrm{C}$ disease is recommended for all children at the age of 12 months, as well as people at high risk of disease. ${ }^{1}$

\section{Measles}

Two cases of measles were notified in NSW in November and none in December. This was a decrease compared to the 77 notifications in September and October 2012. These two cases were the last associated with the measles virus genotype D8 outbreak that began in April. The outbreak was linked to a young traveller who was infected in Thailand.

The first case was a 13-year-old male from metropolitan Sydney with an unknown vaccination history. He was epidemiologically linked to his sister, who had previously been notified with laboratory-confirmed measles (measles virus genotype D8). The second case, the last of the outbreak, was a 14-year-old unvaccinated male from Sydney. The source of infection for this serologicallyconfirmed measles infection remains unknown.

Two doses of measles-mumps-rubella vaccine are recommended for all children (at 12 months and at 4 years of age), ${ }^{1}$ as well as all young adults planning international travel.

\section{Pertussis}

There were 741 pertussis notifications in NSW during the reporting period (430 in November and 311 in December). This is approximately one-third of the 2120 cases notified for the same period in 2011, and represents the lowest number of notifications for this 2-month period since polymerase chain reaction diagnostic testing became widely adopted in 2008. Most cases were in the 5-9-year age group $(n=212)$, followed by the $10-14(n=153)$ and 0 -4-year age groups $(n=117)$.

Direct protection for young infants remains available through free vaccination for pertussis that is administered at 2, 4 and 6 months of age. The first dose can be provided as early as 6 weeks of age. There is also a booster dose at $3 \frac{1}{2}$ to 4 years. New parents and grandparents should also discuss the benefits of pertussis vaccination for themselves with their general practitioner.

\section{Sexually transmissible infections \\ and bloodborne viruses \\ Chlamydia}

After reaching a peak of 2047 notifications in February 2012, the number of monthly chlamydia notifications has decreased. There were 3291 notifications in NSW in November and December 2012, identical to the same period in 2011. A spike in chlamydia notifications is often seen at the beginning of the year, though it was particularly high in 2012.

\section{Gonorrhoea}

There were 635 confirmed cases of gonorrhoea notified in NSW in November and December, an increase of $5.8 \%$ compared to the same period in $2011(n=600)$. The majority of notifications were in males $(n=508)$, and the most commonly notified age group was $20-24$ years $(n=134)$.

\section{Syphilis}

There were 58 syphilis notifications in NSW in November and 30 in December, a decrease since the monthly peak of 78 notifications in July. Delayed reporting may account for some of this decrease.

\section{Lymphogranuloma venereum}

There was an increase in the number of notifications of lymphogranuloma venereum in the second half of 2012. There were 11 notifications in NSW in November and December, which is higher than the same period in 2011 $(n=4)$. Of the 11 notifications, all were male, ranging in age from 18 to 75 years.

\section{Reference}

1. National Health and Medical Research Council. The Australian Immunisation Handbook. 9th ed. Canberra: Australian Government Department of Health and Ageing; 2008. 
Figure 1. Reports of selected communicable diseases, NSW, Jan 2004 to Dec 2012, by month of onset.

Preliminary data: case counts in recent months may increase because of reporting delays.

Laboratory-confirmed cases only, except for measles, meningococcal disease and pertussis.

$\mathrm{BFV}=$ Barmah Forest virus infections, $\mathrm{RRV}=$ Ross River virus infections, lab conf $=$ laboratory

confirmed, Men Gp $C$ and $G p B=$ meningococcal disease due to serogroup $C$ and

serogroup $B$ infection, other/unk = other or unknown serogroups.

NB: Multiple series in graphs are stacked, except gastroenteritis outbreaks.

NB: Outbreaks are more likely to be reported by nursing homes and hospitals than by other institutions.

\begin{tabular}{lr}
\hline \multicolumn{2}{l}{ NSW Population } \\
Male & $50 \%$ \\
$<5$ y & $7 \%$ \\
$5-24$ y & $27 \%$ \\
$25-64$ y & $53 \%$ \\
$65+$ y & $13 \%$ \\
Rural & $46 \%$ \\
\hline
\end{tabular}

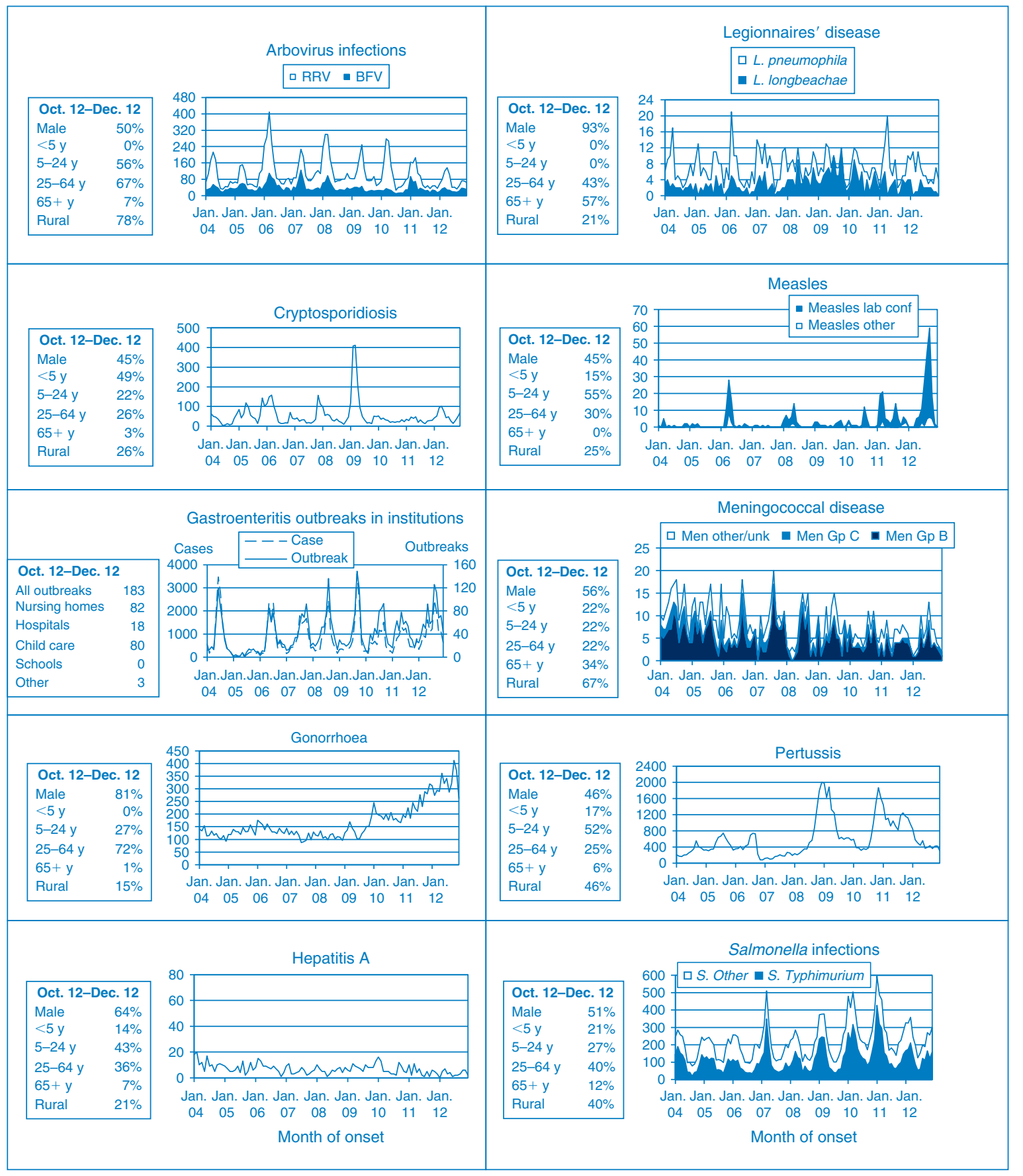




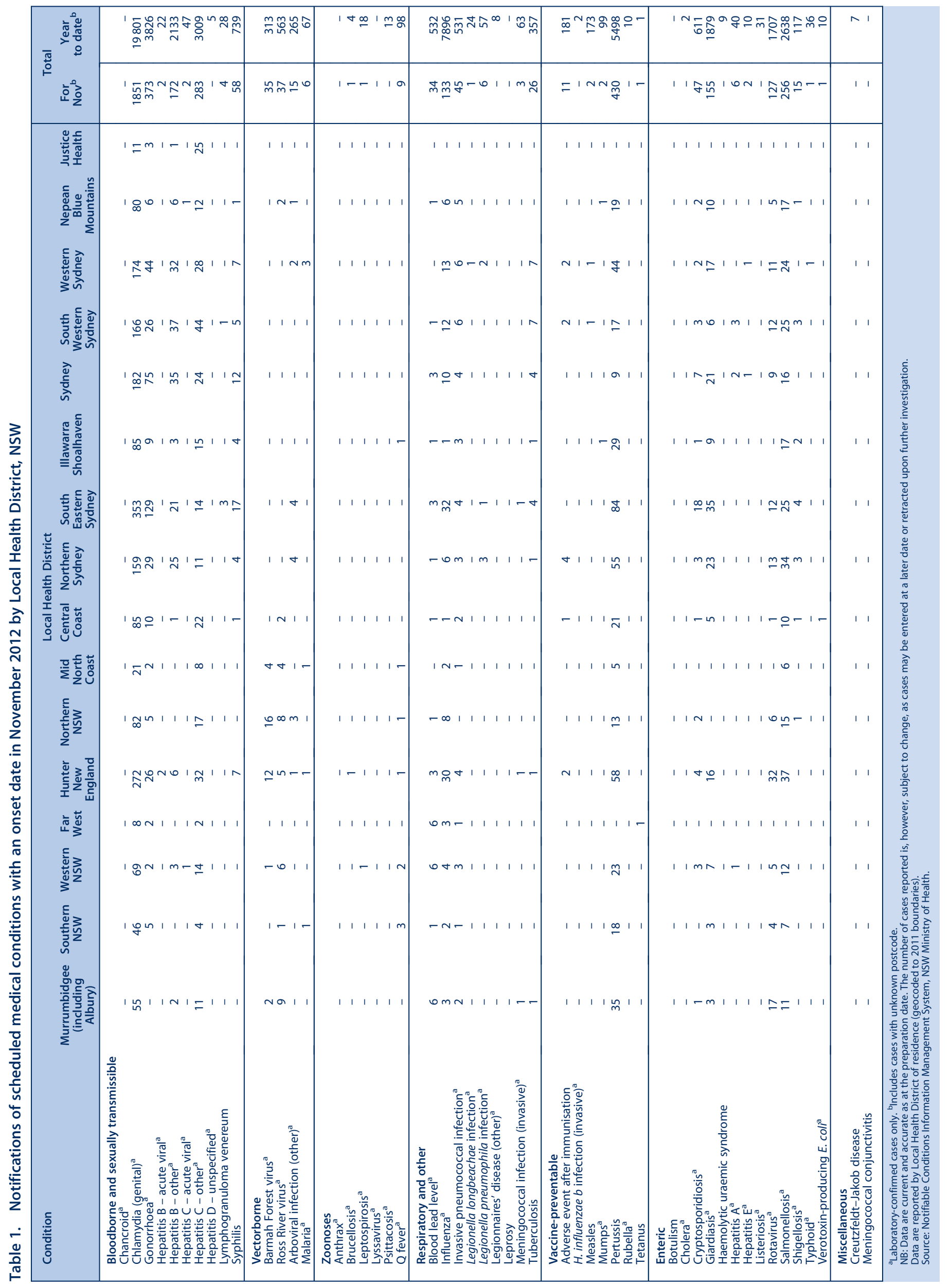




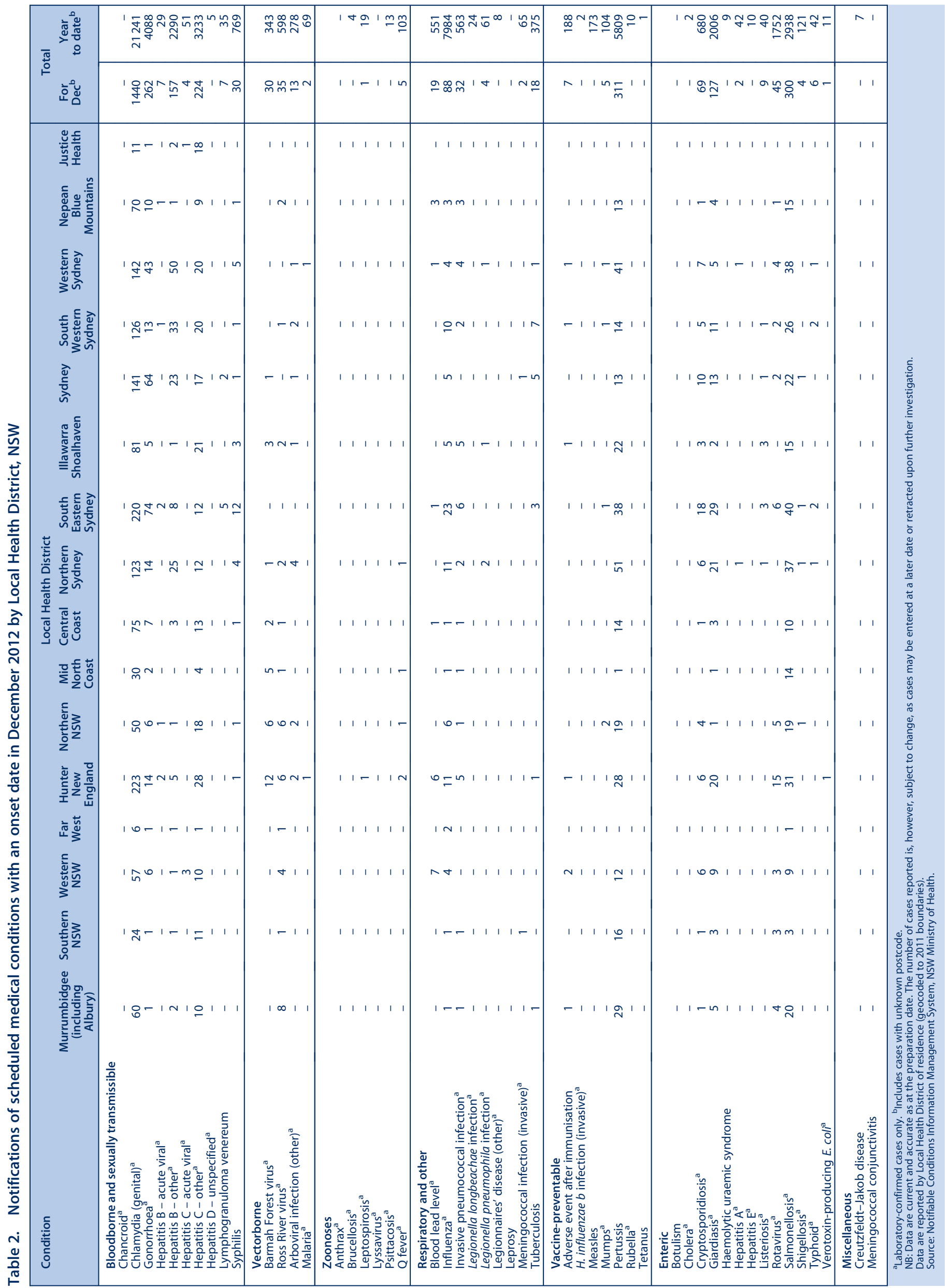

